\title{
Environment, Directed Technical Change
}

\section{and Economic Policy}

André Grimaud ${ }^{1}$ and Luc Rouge ${ }^{2}$

July 2007

${ }^{1}$ Toulouse School of Economics (IDEI and LERNA) and Toulouse Business School

${ }^{2}$ Toulouse Business School 


\begin{abstract}
We study the effects of an economic policy in an endogenous growth general equilibrium framework where production of consumption goods requires two resource inputs: a polluting non-renewable resource and a non-polluting labour resource. The use of the latter contributes to the accumulation of pollution in the atmosphere, which affects welfare. There is a specific research sector associated with each of those resources. We provide a full welfare analysis, and we describe the equilibrium paths in a decentralized economy. We go on to study the effects of three associated economic policy tools: a tax on the polluting resource, and two research subsidies. We show that the optimal environmental policy has two main effects; it delays the extraction of the resource and with it the level of polluting emissions and it reallocates research efforts, decreasing the amount put into "grey" research to the benefit of "green" research. Finally, we compute the optimal values for these tools.
\end{abstract}

Keywords: polluting non-renewable resources, growth, environmental policy, bias of technical change.

JEL classification: O32, O41, Q20, Q32 


\section{Introduction}

It is now common knowledge that a majority of the most serious environmental problems are linked to the use of non-renewable natural resources in production processes. This negative externality raises several problems. These include: which economic policies allow the implementation of optimum, what their impact is on the economy, and in particular on the rate of technical progress? Some of these questions have been addressed in the literature. Basically, we can distinguish two periods.

During the 1990's, most authors dealt with partial equilibrium models. Concerning optimal trajectories, Withagen (1994), in particular, shows that current resource consumption should be lower if pollution is to be taken into account. Hence, extraction has to be postponed. Moreover, Sinclair (1992) shows that an optimal ad valorem tax on the use of non-renewable resources is decreasing. This point is criticized by Ulph \& Ulph (1994), who believe this result is not generally true, particularly in the case of environmental regeneration and extraction costs. Moreover, Hoel \& Kverndokk (1996), who do not consider ad valorem tax, show that the optimal tax increases and then decreases. Finally, some authors, such as Hoel \& Kverndokk (1996) or Tahvonen (1997), consider the possibility of a non-polluting backstop technology. Here, a key issue is the timing of resource use (on this question, see also Chakravorty et al. (1997)). Note that, in these articles, both types of resources are perfect substitutes.

More recently (in the 2000's), problems caused by the use of polluting non-renewable resources have been addressed in the context of general equilibrium models with endogenous growth ${ }^{1}$. Schou (2000 and 2002) studies two kinds of models -human capital, and

\footnotetext{
${ }^{1}$ Several articles consider these questions within the framework of calibrated macroeconomic models: see, for example, Nordhaus and Boyer (2000), Popp (2004), Edenhofer et al. (2005) or Gerlagh and Lise (2005). Moreover, certain authors present analytical or numeric solutions in a partial equilibrium context: in particular, see Liski and Tahvonen (2004). However, few works present a systematic study of the social planner's optimum, the decentralized equilibrium and economic policies in the framework of a general equilibrium model.
} 
R\&D driven growth models- in which no environmental policy is required to implement optimal solutions. Conversely, Grimaud \& Rouge (2005) show in a general model with non-specified functional forms, the utility function in particular, that an environmental policy is generally needed. The optimal (ad valorem) tax is either increasing or decreasing, according to the relative strengths of the evolution over time of pollution's marginal disutility and the psychological discount rate. In the particular case of Schou (2000 \&2002), both effects exactly cancel each other, due to the specified functional forms. Whereas in Schou and Grimaud and Rouge agents, firms or households, are affected by a flow of pollution, Groth and Schou (2006) consider a model in which total factor productivity gradually decreases as a result of the accumulated stock of pollution. This corresponds more to questions associated with the greenhouse effect, and directly follows the partial equilibrium models quoted above.

In this paper, we consider an economy in which two inputs are simultaneously used to produce output: a polluting non-renewable resource, for instance fossil fuels, and a non-polluting input. This second input is produced by means of labour (for a similar type of input, see Smulders and de Nooij (2003)). Here, we are thinking of carbon-free backstop technologies such as solar, and we refer to this input as the labour resource ${ }^{2}$. We have basically three objectives: firstly, to compare the trajectories of the decentralized 'laissezfaire' economy to the optimal ones; second, to study the impact of economic policies, specifically $R \& D$ and climate policies, on the equilibrium variable, namely the path of grey resource extraction, the effort put into the production of the green resource, the effort put into R\&D activities and output growth among others; and finally, to compute the optimal values of the economic policy tools.

To do so, we consider a general equilibrium model with endogenous growth. As we said

\footnotetext{
${ }^{2}$ Popp (2005) uses also a model in which total energy is simultaneously produced by fossil fuels and a carbon-free backstop technology. In his model, this latter input is produced by means of output.
} 
above, two kinds of resources are used within the production process. The use of the nonrenewable resource (the 'grey' resource) yields a flow of pollution which accumulates in the atmosphere. As Groth and Schou (2007) do, we consider pollution as a stock, which could, for example, be green-house gases. This affects the quality of the environment, which here is a variable in households' utility. The other resource (the 'green' resource) is in an alternative to fossil fuels as we have already mentioned (see for instance Hoel and Kverndokk (1996), Tahvonen (1997) or Tahvonen and Salo (2001) on this point); however, this resource does not constitute a perfect substitute, and it is used simultaneously. Moreover we assume that a specific R\&D sector and a specific stock of knowledge are associated with each of these resources. To do so, we follow Acemoglu's work on directed technical change (e.g. 2002), as has been done elsewhere in recent literature. Smulders and de Nooij (2003), as well as Andre and Smulders (2004), for instance, introduce this type of analysis in an endogenous growth model, but they do not take pollution into account. Hart (2004) also studies an endogenous growth model without non-renewable resources, but where pollution can be reduced by a type of research which is environmentally oriented, contrary to a second type of research, which is labelled 'ordinary'. Thus we will have two stocks of knowledge. One is associated with the polluting non-renewable resource ('grey' knowledge), and the other is associated with the clean labour resource ('green' knowledge); we refer to the relative evolution of the two stocks of knowledge as the direction of technical change (as in Acemoglu (2002) for instance). Hence, studying the impact of economic policies on $\mathrm{R} \& \mathrm{D}$ leads us to distinguish the effects on the total amount of research ('quantity' of research), and also on the allocation of research inputs between research sectors ('quality' of research) (see also Hart (2004)).

Another key feature of our model is that innovations are not embodied in intermediate goods, as, for instance, in Gerlagh and Lise (2005), Grimaud and Rouge (2004 and 2005), 
or Popp (2006). Here, we assume that knowledge is directly priced. This allows us to considerably simplify the calculations within this type of model. In particular, this enables to conduct a welfare analysis, which is generally not done in models with directed technical change and intermediate goods (see for instance Acemoglu (2002)). Moreover, in standard endogenous growth models with intermediate goods, implementation of the optimum requires two tools aimed at correcting both distortions that stem from aspects of the structure of research market, namely monopoly power and intertemporal spillover (see Barro \& Sala-i-Martin (2005)). If environmental externalities are added, the problem becomes very complicated, as is also discussed in Gerlagh \& Lise (2005).

In this paper, we first determine the optimal paths. We provide a complete characterization of the dynamics of all variables in the economy; in fact, we study the transition towards the steady-state. In particular, we describe the optimal resource extraction path, thus determining the path of pollution accumulation in the atmosphere. We simultaneously establish the optimal allocation of effort put into each of the two research sectors.

We go on to study the positive aspects of our analysis through the economy's decentralized equilibrium properties, which we compare to the optimal ones. The absence of intermediate goods in the economy leads us to define a decentralized equilibrium which departs from the standard in the endogenous growth context: because of the non-convexity of technology, we assume that firms compete in Cournot fashion in markets for consumption goods.

At the equilibrium, there are three fundamental distortions: the environmental externality presented above and two externalities arising from the fact that in each research sector innovators cannot extract the whole surplus from users of innovations (on this point, see Jones \& Williams (1998) or Popp (2006) for instance). Following Tinbergen (1960), we thus associate three economic policy tools: an ad valorem tax on the use of the grey 
resource, and two subsidies for both research sectors. Hence, the equilibrium variables (quantities, prices and their growth rates) are functions of these economic policy instruments. We consider the effects of the policies on the equilibrium variables and we compute the optimal values for these economic policy tools.

The main results of the paper are the following.

First, we show that there exists one stable unique feasible optimal steady-state. Optimal variables tend towards this regime, which corresponds to the optimum in the case where there is no pollution, or, equally, pollution does not affect welfare. This comes from the fact that, as the stock of non-renewable resource is fully exhausted in infinite time, the extraction and pollution flows tend to zero asymptotically.

Second, when we compare the optimum and the 'laissez-faire' regimes, we show that the decentralized economy uses the non-renewable resource too fast, and thus too much pollution is emitted in the early stages of the process. This confirms a result of Withagen (1994) who considers a partial equilibrium model in which the stock of pollutants decays at a constant exogenous rate. At the same time, the equilibrium quantity of research, that is, the overall research effort, is sub-optimal. Moreover, the effort invested in green research is always too low, whilst the effort in grey research is too high in the early stages. It is important to note that this situation in grey research is reversed after a certain period. The length of this period is inversely correlated to the distortion in the innovation market, that is, the gap between the price paid by users of an innovation and their marginal willingness to pay. We also show that the direction of technical change, measured here as the difference between the growth rates of 'green' and 'grey' resource stocks (referring to Acemoglu (2002)), is non optimal as it is too 'grey-oriented'. Finally, decentralized equilibrium growth is sub-optimal; which means that early generations consume too much to the detriment of the future generations. 
Third, we determine the effects of the two economic policies. The R\&D policy promotes both types of research effort (green and grey): the quantity and the quality of research increase. However, the direction of technical change remains unchanged. We also show that the flows of extraction (and thus of pollution) are also unchanged, as are the dynamics of the environment. Concerning the effects of the optimal environmental policy, our first set of results confirms standard findings from the previous literature. We show that the level of tax does not matter, only resulting in rent transfers (as in Sinclair (1992), Grimaud Rouge (2005) and Groth Schou (2007) for instance). The optimal climate policy, which is shown to levy a decreasing tax on fossil fuels, will hold back the pace of extraction, and thus slow down polluting emissions. A simple intuition is that the price of the resource (including the tax) becomes relatively higher today. Furthermore, we demonstrate that, as growth rates of resource extraction and green knowledge are increased, this policy fosters output growth. More precisely, in our model the level of output is lower for early generations and higher for future ones, as resource extraction is postponed (see Grimaud Rouge (2005) for a similar result). We show that this results in a loss of welfare for early generations. The second set of results concerns the impact of the optimal climate policy on the overall R\&D effort and the direction of technical change. It is shown that the quantity of research is not modified. However, the quality of research is modified: the effort put into 'grey' research decreases, thus benefiting 'green' research. In other words, this decreasing environmental tax steers technical change in the 'desired' direction. This result has to be linked to Andre and Smulders (2004) who show, in a model without pollution, that a decrease in the growth rate of a tax on the non-renewable resource shifts research activity from "energy-related" to "labour-related" knowledge. Furthermore, we study the impact of the climate policy on the ratio of green and grey resources' marginal productivities, which we refer to as the bias of technical change (following Acemoglu (2002)). We show 
that the environmental policy is grey-biased in the short-term, and green-biased in the long-term.

We conclude by determining the optimal values of the economic policy instruments.

Section 2 introduces the model and presents welfare analysis. The equilibrium of the decentralised economy is studied in Section 3. The effects of economic policies and the calculation of optimal policy tools are presented in Section 4. Finally, in section 5, we make some concluding remarks.

\section{Model and welfare}

\subsection{Model}

There is a continuum of consumption goods, indexed on the unit interval. Each good $j$, $j \in[0 ; 1]$, is produced by $N_{j}$ firms. Each firm $n_{j}\left(n_{j}=1, \ldots, N_{j}\right)$ simultaneously produces good $j$ and performs research. For firm $n_{j}$, production function of good $j$ is

$$
Y_{n_{j} t}=\left[\beta\left(A_{Q t} Q_{n_{j} t}\right)^{\alpha}+(1-\beta)\left(A_{R t} R_{n_{j} t}\right)^{\alpha}\right]^{1 / \alpha},-\infty<\alpha \leq 1 \text { and } \beta \in(0 ; 1) .
$$

$R_{n_{j t}}$ is the flow of non-renewable resource. The input simultaneously used within the production process, $Q_{n_{j t}}$, is produced from labour, as it is mentioned later in the text (see formula (4)). Hence we refer to it as the labour resource. In that sense, this production function is very similar to those used in Smulders \& de Nooij (2003) or Andre \& Smulders (2004).

$A_{Q t}$ and $A_{R t}$ are the stocks of specific knowledge for the two resources. In fact, the combined use of both resources can be interpreted from the fact that they are imperfect substitutes (that are used in specific niche markets, see for instance Gerlagh and van der Zwaan (2003) on this point). 
This CES technology is such that there is some complementarity between each resource and its associated knowledge. Indeed, since $\alpha$ is lower than $1, \partial Y / \partial A_{Q}$ and $\partial Y / \partial A_{R}$ are increasing functions of $Q$ and $R$ respectively: marginal productivity for any stock of knowledge is increasing with the use of the associated resource. This is a key assumption when studying the impact of climate policiy on the direction of technical change (see section 4.1.2).

Note that $\sigma=1 /(1-\alpha)$ is the elasticity of substitution between the two factors. Since $-\infty<\alpha \leq 1, \sigma$ is positive. Then, following Dasgupta and Heal (1979, p.197), the non-renewable resource is necessary, that is, $Y_{n_{j} t}=0$ if $R_{n_{j} t}=0$, when $0 \leq \sigma \leq 1$, i.e. $-\infty<\alpha \leq 0$. When $1<\sigma<+\infty$, i.e. $0<\alpha<1$, it is non-necessary. If $\alpha$ tends to 1 (i.e. $\sigma$ tends to $+\infty$ ), the production function is linear; when $\alpha=0$ (i.e. $\sigma=1$ ), the production function is Cobb-Douglas; when $\alpha$ tends to $-\infty$ (i.e. $\sigma$ tends to 0 ), it is Leontieff.

Technologies for production of knowledge are

$$
\dot{A}_{Q n_{j} t}=\delta_{Q} L_{Q n_{j} t} A_{Q t}, \delta_{Q}>0,
$$

and

$$
\dot{A}_{R n_{j} t}=\delta_{R} L_{R n_{j} t} A_{R t}, \delta_{R}>0
$$

$A_{Q n_{j} t}$ and $A_{R n_{j} t}$ are the stocks of knowledge produced by firm $n_{j}$, and we have $A_{Q t}=$ $\int_{0}^{1}\left(\sum_{n_{j}}^{N_{j}} A_{Q n_{j} t}\right) d j$ and $A_{R t}=\int_{0}^{1}\left(\sum_{n_{j}}^{N_{j}} A_{R n_{j} t}\right) d j$.

The flow $Q_{t}$ of labour resource is produced with a quantity $l_{t}$ of labour:

$$
Q_{t}=\theta l_{t}, \theta>0
$$

The non-renewable resource is extracted from an initial finite stock $S_{0}$. Extraction costs 
are modelled following Andre and Smulders (2004). At each date $t$, a flow $-\dot{S}_{t}$ of nonrenewable resource is extracted, and a proportion

$$
R_{t}=-\dot{S}_{t} /\left(1+\mu_{t}\right), \mu_{t}>0
$$

is supplied on the market, while $-\dot{S}_{t} \mu_{t} /\left(1+\mu_{t}\right)$ vanishes. $\mu_{t} /\left(1+\mu_{t}\right)$ is the unit cost of extraction in terms of resource. We will later on denote by $\hat{\mu}_{t}$ the term $\dot{\mu}_{t} /\left(1+\mu_{t}\right)$. If $\hat{\mu}_{t}<0$, the unit cost of extraction is decreasing over time, because of technical progress that increases exploration efficiency. Conversely, $\hat{\mu}_{t}$ can be positive if we consider that exploitable reserves are getting less accessible despite better drilling results.

The labour resource does not pollute. Pollution is generated by the use of the nonrenewable natural resource within the production process:

$$
P_{t}=\gamma R_{t}, \gamma>0
$$

This flow of pollution $\left(P_{t}\right)$ affects negatively the stock of environment $\left(E_{t}\right)$. We assume $E_{t}=E_{0}-\int_{0}^{t} P_{s} d_{s}, E_{0}>0$, which gives the following law of motion

$$
\dot{E}_{t}=-P_{t}=-\gamma R_{t}
$$

In the following, we assume that the lower limit to the stock of environment, $E_{0}-\gamma S_{0}$, is positive. We show below that the resource is asymptotically exhausted, and thus that the stock of environment tends to its lower limit in the long-run. Hence, since the total quantity of pollution emitted in the atmosphere is known, the question is, what is the pollution path, or, in other words, how will pollution be distributed between generations (as in Grimaud and Rouge (2005)). Note that we do not consider environmental regeneration 
(as it is done in a similar context by Groth and Schou (2007)). If this were to feature in the model, as well as abatement activities ${ }^{3}$, it would certainly affect the results. Considering carbon sequestration, in particular, would lead to a dissociation of the pollution flow and extraction.

Population is assumed constant, normalized to one, and each individual is endowed with one unit of labour. Thus we have:

$$
1=l_{t}+L_{Q t}+L_{R t}
$$

where $l_{t}$ is used for production and $\left(L_{Q t}+L_{R t}\right)$ for research. Note that $L_{Q t}=\int_{0}^{1}\left(\sum_{n_{j}}^{N_{j}} L_{Q n_{j} t}\right) d j$ and $L_{R t}=\int_{0}^{1}\left(\sum_{n_{j}}^{N_{j}} L_{R n_{j} t}\right) d j$.

The household's instantaneous utility function depends both on consumption $c_{j t}, j \in$ $[0 ; 1]$, and the stock of environment $E_{t}$. The intertemporal utility function is:

$$
U=\int_{0}^{+\infty}\left[\ln \left(\int_{0}^{1} c_{j t}^{\varepsilon} d j\right)^{1 / \varepsilon}+\omega \ln E_{t}\right] e^{-\rho t} d t, 0<\varepsilon<1, \rho>0 \text { and } \omega>0 .
$$

where $c_{j t}=Y_{j t}=\sum_{n_{j}}^{N_{j}} Y_{n_{j}}$, that is, the whole production of good $j$ is consumed by the representative household.

\subsection{Welfare}

We now characterize the optimum, that is, the solution of the social planner's program (we give more details in Appendix 1). Moreover, we consider the symmetric case in which consumption good sectors and firms are identical. In this case, we have $N_{j}=N$, $Y_{n_{j}}=Y / N, Q_{n_{j}}=Q / N, R_{n_{j}}=R / N, L_{Q n_{j}}=L_{Q} / N$, and $L_{R n_{j}}=L_{R} / N$. The results are given in proposition $1^{4}$; using these results and the phase diagram given in figure 1 ,

\footnotetext{
${ }^{3}$ We thank one anonymous referee for this remark.

${ }^{4}$ Proposition 1 only features the growth rates of $R, E, Y, A_{Q}$ and $A_{R}$. If we look for analytical solutions, the only way to compute the optimal levels of these variables, that is, the functions of time $R(t), E(t)$,
} 
we fully characterize the optimal transitional dynamics of the economy. We drop time subscripts for notational convenience.

Proposition 1 At the social optimum, quantities and rates of growth take the following values (upper-script ${ }^{o}$ is used for optimum, and $g_{X}$ is the rate of growth of any variable $X)$ :

$$
\begin{gathered}
l^{o}=\frac{\rho}{\delta_{Q}}, Q^{o}=\theta l^{o}, \\
L_{Q}^{o}=\frac{\delta_{R}\left(\delta_{Q}-\rho\right)}{\delta_{Q}\left(\delta_{Q}+\delta_{R}\right)}+\frac{g_{R}^{o}}{\delta_{Q}+\delta_{R}}, \\
L_{R}^{o}=\frac{\delta_{Q}-\rho}{\delta_{Q}+\delta_{R}}-\frac{g_{R}^{o}}{\delta_{Q}+\delta_{R}}, \\
g_{R}^{o}=-\rho-\hat{\mu}\left(1-\frac{\gamma\left(\delta_{Q}+\delta_{R}\right) R}{\delta_{Q}} \int_{t}^{+\infty}(\omega / E) e^{-\rho(s-t)} d s\right)-\frac{\omega\left(\delta_{Q}+\delta_{R}\right)}{\delta_{Q}} g_{E}^{o}, \\
g_{Y}^{o}=\delta_{Q} L_{Q}^{o} . \\
g_{A_{Q}}^{o}=\delta_{Q} L_{Q}^{o}, g_{A_{R}}^{o}=\delta_{R} L_{R}^{o}, \text { and } g_{A_{Q}}^{o}-g_{A_{R}}^{o}=g_{R}^{o} .
\end{gathered}
$$

Proof. See Appendix 1.

First of all, note that, if $\omega=0$, which corresponds to the case where households are indifferent to the state of environment, the economy immediately jumps to its steady-state (that is, an economy in which all rates of growth are constant). Indeed, in this case we have $g_{R}^{o}=-\rho-\hat{\mu}($ see $(13))$, which is constant if we assume that $\hat{\mu}$ is constant. Thus, transitional dynamics of the model stem from the introduction of the stock of environment $\left(E_{t}\right)$.

$Y(t)$ among others, would be to use the initial conditions $E(0)=E_{0}$ and $S(0)=S_{0}$ (where $E_{0}$ and $S_{0}$ are given positive constants) and the condition $S_{0}=\int_{0}^{+\infty}\left(1+\mu_{t}\right) R_{t}^{o} d t$. It is very difficult (maybe impossible) to compute such solutions, in particular because of the complexity of the differential equation giving the optimal growth rate of resource extraction. However, we think that the phase diagram (Figure 1) together with the trajectories presented in Figure 2 give several indications on these optimal variables. One possible way to go further in the analysis would be to perform a numerical analysis. 
Let us now study the dynamics of the optimum, in the particular case where $\hat{\mu}=0$ (i.e. constant extraction costs) for computational convenience.

From (7), $\dot{E}^{o}=-\gamma R^{o}$, one gets $g_{E}^{o}=-\gamma R^{o} / E^{o}$. Log-differentiating with respect to time, we have $\dot{g}_{E}^{o} / g_{E}^{o}=g_{R}^{o}-g_{E}^{o}$, or $\dot{g}_{E}^{o}=g_{E}^{o}\left(g_{R}^{o}-g_{E}^{o}\right)$. Let us study the evolution of the environment's growth rate over time. Plugging (13) (where $\hat{\mu}=0)$ into $\dot{g}_{E}^{o}=g_{E}^{o}\left(g_{R}^{o}-g_{E}^{o}\right)$ gives the following Ricatti differential equation : $\dot{g}_{E}^{o}=-\left(1+\omega\left(\delta_{Q}+\delta_{R}\right) / \delta_{Q}\right)\left(g_{E}^{o}\right)^{2}-\rho g_{E}^{o}$. In order to transform this equation into a linear first-order differential equation, we consider the new variable $z=1 / g_{E}^{o}$, which implies $\dot{z}=-\dot{g}_{E}^{o} /\left(g_{E}^{o}\right)^{2}$. The Ricatti equation becomes $\dot{z}=\left(1+\omega\left(\delta_{Q}+\delta_{R}\right) / \delta_{Q}\right)+\rho z$, whose solution leads to

$$
g_{E}^{o}=\frac{1}{e^{\rho t}\left[1 / g_{E 0}^{o}+\left(\delta_{Q}+\omega\left(\delta_{Q}+\delta_{R}\right)\right) / \rho \delta_{Q}\right]-\left(\delta_{Q}+\omega\left(\delta_{Q}+\delta_{R}\right)\right) / \rho \delta_{Q}} .
$$

In order to construct a phase diagram, recall that we have $g_{R}^{o}=-\rho-\left(\omega\left(\delta_{Q}+\delta_{R}\right) / \delta_{Q}\right) g_{E}^{o}$ (see (13) when $\hat{\mu}=0)$. Moreover, from $\dot{g}_{E}^{o}=g_{E}^{o}\left(g_{R}^{o}-g_{E}^{o}\right)$ (which we obtained above), we get $\dot{g}_{E}^{o} \leq 0$ and $\dot{g}_{R}^{o} \geq 0$ if $g_{R}^{o} \geq g_{E}^{o}$. Similarly, $\dot{g}_{E}^{o} \geq 0$ and $\dot{g}_{R}^{o} \leq 0$ if $g_{R}^{o} \leq g_{E}^{o}$.

Before studying this phase diagram, we need to make two preliminary remarks. First, the flow of extraction is strictly positive at each date $t$. Indeed, if $\alpha \leq 0$, that is, $\sigma \leq 1$, the resource is necessary, i.e. output is nil when $R=0$. If $\alpha>0$, that is, $\sigma>1$, the resource is non-necessary, but $\lim _{R \rightarrow 0} \partial Y / \partial R=+\infty$. This means that the conditions for proposition 5 ' in Dasgupta and Heal (1974, footnote 1, page 15) to hold are fulfilled; hence $R_{t}>0$ for all $t \geq 0$.

Second, the stock of non-renewable resource is fully depleted. This is shown by the following. At each date $t$, extracting one unit of grey resource has two effects; production, and thus consumption increase, but at the same time the environment is harmed. Therefore utility is affected in two opposite ways: the first effect increases the current level of utility, whereas the second diminishes utility levels from $t$ to infinity. One can verify that 
the first effect, $\left(\partial Y_{t} / \partial R_{t}\right) / Y_{t}$, tends to infinity as $R_{t}$ tends to zero, whereas the second one is bounded ${ }^{5}$. Indeed, the marginal disutility of resource extraction (and thus pollution) is bounded since the level of the environment is bounded. Formally, this effect is given by $\int_{t}^{+\infty}\left(\omega / E_{s}\right)\left(d E_{s} / d R_{t}\right) e^{-\rho(s-t)} d s$, where $d E_{s} / d R_{t}=-\gamma$, and $1 / E_{s}<1 /\left(E_{0}-\gamma S_{0}\right)$ since $E_{0}-\gamma S_{0}$ is the positive lower limit of the environment, reached when the resource is fully depleted. Finally, we get $0>\int_{t}^{+\infty}\left(\omega / E_{s}\right)\left(d E_{s} / d R_{t}\right) e^{-\rho(s-t)} d s>-\gamma \omega / \rho\left(E_{0}-\gamma S_{0}\right)$. These formulae are the ones presented in Appendix 1 (see (33) when $\varphi_{S}=0$ ).

Let us now study the phase diagram depicted in figure 1. There are two steady-states. The first one is unstable, and it occurs when $g_{E 0}^{o}=g_{R 0}^{o}=-\rho \delta_{Q} /\left(\delta_{Q}+\omega\left(\delta_{Q}+\delta_{R}\right)\right)$ : this corresponds to $\mathrm{J}$ in the phase diagram. Along this steady-state, $\dot{E}$ remains strictly positive as $t$ tends to infinity. This is impossible, since $\lim _{t \rightarrow+\infty} R_{t}=0$. Therefore, this steady-state can be ruled out ${ }^{6}$. In addition, we can also eliminate the path JK. Indeed, since $g_{R}$ is alternately negative and positive along this path, the stock of resource is fully exhausted in finite time. This contradicts what is stated above.

The second steady-state, I in figure 1, is stable, and the economy tends towards it if $g_{E 0}^{o}>-\rho \delta_{Q} /\left(\delta_{Q}+\omega\left(\delta_{Q}+\delta_{R}\right)\right)$. This limit regime corresponds to the case in which optimal extraction $R^{o}$, and thus optimal pollution, $P^{o}=\gamma R^{o}$, tend to zero. Hence $\dot{E}^{o}$ also tends to zero. This corresponds to the "laisser-faire" case (see section 3).

Let us now give some details about the transition towards this regime. Our first comments are based on Figure 1. We can see that the growth rate of resource extraction, $g_{R}^{o}$, is unambiguously negative. Moreover this rate decreases over time and asymptotically tends towards its lower limit $-\rho$ (which is the level of this rate in the case of no pollution). This means that, though this difference decreases over time, the optimal growth rate of

\footnotetext{
${ }^{5}$ In some cases, the resource may not be fully depleted. For instance, if it is non-necessary for production and if its marginal productivity is bounded from above (on this point, see for instance Gerlagh and Keyzer (2004)).

${ }^{6}$ We thank one anonymous referee for this remark.
} 
resource extraction is always higher than the "no-pollution" one. Thus, the fact that the production process pollutes and affects welfare implies that it is optimal to postpone resource extraction. That is less grey resource is used today, and more tomorrow. This results confirms the findings of Withagen (1994, p.241) in a partial equilibrium framework with no growth.

On the other hand, the optimal growth rate of the stock of environment, $g_{E}^{o}$, which is also unambiguously negative (see (7)), increases over time and tends towards zero, its obvious upper limit. Indeed, the flow of pollution becomes nil in a distant future, as the grey resource gets exhausted. For this reason, the state of the environment decays more and more slowly.

Proposition 1 shows that the dynamics of $g_{R}^{o}$ and $g_{E}^{o}$ have a direct impact on the flows of labour devoted to research and the growth rate of the economy. From (11) and (12), we can see that pollution leads the social planner to devote more effort to green research $\left(L_{Q}^{o}\right)$, and less to grey research $\left(\mathrm{L}_{R}^{o}\right)$. Along the transitional path, $L_{Q}^{o}$ decreases and $L_{R}^{o}$ increases, both converging towards their "no-pollution" levels. At the same time, equation (14) shows that the economy's growth rate is higher in the pollution case, as $g_{R}^{o}$ is also higher (see above). Then $g_{Y}^{o}$ decreases over time, to eventually converge towards its no-pollution level.

\section{display Figure 1 here}

Dynamics of the optimal (social planner's program) and decentralized equilibrium paths are fully depicted in Figure 2: see section 3.2. 


\section{Equilibrium in the decentralized economy}

Let us now turn to the decentralized economy, and in particular the way we model innovation activities.

In contrast with the standard endogenous growth literature, in our model new pieces of knowledge are not embedded in intermediate goods. They are directly used by firms and protected by infinitely-lived patents (that is, directly priced). As knowledge is a public good, there are two main difficulties in funding it. First, it is difficult to extract from agents their total willingness to pay for the use of that knowledge (see for instance Popp (2004)); according to Jones and Williams (1998), investments in R\&D in the US are at least two to four times lower than their optimal level. We therefore introduce two exogenous parameters $\psi_{Q}$ and $\psi_{R}$ (see section 3.2), which represent the gap between the willingness to pay and the price of innovations in both research sectors as received by sellers (these parameters will be interpreted as subsidies to $R \& D$ later in the text). A second difficulty arises because the technologies of firms using knowledge as a productive factor are nonconvex (see formulas (1), (2) and (3)). In a perfectly competitive environment, profits for these firms would be negative and a general competitive equilibrium would not exist. We therefore assume imperfect competition (à la Cournot) in markets for consumption goods. By selling these goods at a price which is higher than the marginal cost, firms gain resources that allow them to buy knowledge.

There are four basic distortions with respect to the social planner's program. First, the flow of pollution, $P_{t}$, which damages the stock of environment; second, the two distortions in markets for innovations mentioned above; and, finally, the Cournot competition in the markets for consumption goods. This latter distortion will be shown not to prevent decentralized equilibrium variables to be optimal (see section 3.2.2 below). Hence we introduce three economic tools: a tax on the polluting resource, and two subsidies to 
research.

\subsection{Agents' behaviour}

Wage is normalized to one: $w_{t}=1$, and $p_{j t}, p_{Q t}, p_{R t}$ and $r_{t}$ are, respectively, the price of consumption good $j$, the price of the labour and non-renewable resources, and the interest rate on a perfect financial market. We drop time subscripts for notational convenience.

\section{Household}

The representative household maximizes (9) subject to her budget constraint $\dot{b}=$ $r b+w+\pi-\int_{0}^{1} p_{j} c_{j} d j+T$, where $b$ is her total wealth, $\pi$ represents total profits in the economy and $T$ is a lump-sum subsidy (or tax). Recall that we normalized $w$ to 1 . One gets the two following standard results (details are given in Appendix 2). Total demand for $\operatorname{good} j$ is

$$
c_{j}=p_{j}^{1 /(\varepsilon-1)} \Omega,
$$

where $\Omega=\left(\int_{0}^{1} p_{k} c_{k} d k\right) /\left(\int_{0}^{1} p_{k}^{\varepsilon /(\varepsilon-1)} d k\right)$, and Ramsey-Keynes condition is

$$
r=\rho+(1-\varepsilon) g_{c_{j}}+g_{\Gamma}+g_{p_{j}}, \text { with } j \in[0 ; 1]
$$

where $\Gamma=\int_{0}^{1} c_{j}^{\varepsilon} d j$.

\section{Labour resource sector:}

The profit of the firm is $\pi^{Q}=p_{Q} \theta l-l$. Perfect competition leads to

$$
p_{Q}=1 / \theta
$$

\section{Non-renewable resource sector:}

On the competitive natural resource market, the maximization of the profit function $\int_{t}^{+\infty} p_{R s} R_{s} e^{-\int_{t}^{s} r_{u} d u} d s$, subject to $\dot{S}_{s}=-\left(1+\mu_{s}\right) R_{s}, S_{s} \geq 0, R_{s} \geq 0, s \geq t$, yields the 
standard equilibrium "Hotelling rule":

$$
\frac{\dot{p}_{R}}{p_{R}}=r+\hat{\mu}
$$

Equation (20) states that the owner's net rent, $\dot{p}_{R} / p_{R}-\hat{\mu}$, is equal to the interest rate. In particular, note that if $\hat{\mu}<0$ (if technical progress increases access to exploitable resource stocks) one gets $\dot{p}_{R} / p_{R}<r$. The case where $\dot{p}_{R} / p_{R}<0$ can even occur (if the decrease in extraction costs is fast enough). As usual, the transversality condition is $\lim _{t \rightarrow+\infty} S_{t}=0$.

\section{Firms}

Recall that firms have two activities: first, each one produces and sells a differentiated good on an imperfect market. Second, it produces and sells innovations which we assume traded using bilateral contracts between inventors and users.

$V_{Q t}$ and $V_{R t}$ are the prices of one innovation at date $t$ in the two research sectors. Let us denote by $\tilde{\pi}_{n_{j} t}$ profit of firm $n_{j}$ without payment of knowledge. At each moment, firm $n_{j}$ $\operatorname{maximizes} \tilde{\pi}_{n_{j} t}=p_{j t} Y_{n_{j} t}-p_{Q t} Q_{n_{j} t}-\left(1+\sigma_{t}\right) p_{R t} R_{n_{j} t}+V_{Q t} \dot{A}_{Q n_{j} t}+V_{R t} \dot{A}_{R n_{j} t}-L_{Q n_{j} t}-L_{R n_{j} t}$, subject to (1), (2), (3) and (17), where $\sigma_{t}$ is the unit tax on resource use. Henceforth, we will denote $\tau_{t}=1+\sigma_{t}$ for computational convenience. After substitutions, one gets the following program:

$$
\begin{aligned}
\max \tilde{\pi}_{n_{j}}= & Y_{n_{j}}\left[\Omega^{1-\varepsilon}\left(\sum_{n_{k}=1}^{N_{j}} Y_{n_{k}}\right)^{\varepsilon-1}\right]-p_{Q} Q_{n_{j}}-\tau p_{R} R_{n_{j}}+V_{Q} \delta_{Q} L_{Q n_{j}} A_{Q} \\
& +V_{R} \delta_{R} L_{R n_{j}} A_{R}-L_{Q n_{j}}-L_{R n_{j}} \\
\text { subject to } Y_{n_{j}}= & {\left[\beta\left(A_{Q} Q_{n_{j}}\right)^{\alpha}+(1-\beta)\left(A_{R} R_{n_{j}}\right)^{a}\right]^{1 / \alpha}, }
\end{aligned}
$$

The first order conditions with respect to $Y_{n_{j}}, Q_{n_{j}}, R_{n_{j}}, L_{Q n_{j}}$, and $L_{R n_{j}}$ are respect- 
ively ( $\lambda$ is the Lagrange multiplier):

$$
\Omega^{1-\varepsilon}\left(\sum_{n_{k}=1}^{N_{j}} Y_{n_{k}}\right)^{\varepsilon-1}+(\varepsilon-1) Y_{n_{j}} \Omega^{1-\varepsilon}\left(\sum_{n_{k}=1}^{N_{j}} Y_{n_{k}}\right)^{\varepsilon-2}=\lambda .
$$

This equation implicitely yields the best response of firm $n_{j}$ to the choice of production of the other firms on the market of consumption good $j$.

$$
\begin{gathered}
p_{Q}=\lambda \beta Y_{n_{j}}^{1-\alpha} A_{Q}^{\alpha} Q_{n_{j}}^{\alpha-1} \\
\tau p_{R}=\lambda(1-\beta) Y_{n_{j}}^{1-\alpha} A_{R}^{\alpha} R_{n_{j}}^{\alpha-1} \\
V_{Q} \delta_{Q} A_{Q}=1 \\
V_{R} \delta_{R} A_{R}=1 .
\end{gathered}
$$

The willingnesses to pay for pieces of knowledges $A_{Q}$ and $A_{R}$ at each date $t$ respectively are

$$
v_{Q n_{j}}=\partial \tilde{\pi}_{n_{j}} / \partial A_{Q}=V_{Q} \delta_{Q} L_{Q n_{j}}+\lambda \beta Y_{n_{j}}^{1-\alpha} A_{Q}^{\alpha-1} Q_{n_{j}}^{\alpha}
$$

and

$$
v_{R n_{j}}=\partial \tilde{\pi}_{n_{j}} / \partial A_{R}=V_{R} \delta_{R} L_{R n_{j}}+\lambda(1-\beta) Y_{n_{j}}^{1-\alpha} A_{R}^{\alpha-1} R_{n_{j}}^{\alpha} .
$$

Both formulas are composed of two parts. Each piece of knowledge being simultaneously used by research and production activities, these parts correspond to the respective willingnesses to pay. We recover, here, the public good nature of knowledge inside the firm.

\subsection{Decentralized equilibrium}

Here, an equilibrium is a set of profiles of quantities and prices, such that: the representative household maximizes utiliy and firms maximize profits; labour, resource and financial 
markets are perfectly competitive; on each consumption good market, there is Cournot competition; pieces of knowledge are traded using bilateral contracts. We focus on a symmetric decentralized equilibrium (where, as in section 2.2, consumption good sectors and firms are identical).

\subsubsection{Characterization of the decentralized equilibrium}

From (22) and (26) we get $v_{Q n_{j}}=V_{Q} \delta_{Q} L_{Q n_{j}}+p_{Q} Q_{n j} / A_{Q}=V_{Q} \delta_{Q} L_{Q n_{j}}+l_{n j} / A_{Q}$. We also obtain from (23) and (27) $v_{R n_{j}}=V_{R} \delta_{R} L_{R n_{j}}+\tau p_{R} R_{n j} / A_{R}$. Summing on $n_{j}$ and $j$, we get the total willingness to pay for one unit of "green" knowledge at date $t, v_{Q}=$ $V_{Q} \delta_{Q} L_{Q}+l / A_{Q}$, and the total willingness to pay for grey knowledge at date $t, v_{R}=$ $V_{R} \delta_{R} L_{R}+\tau p_{R} R / A_{R}$. These correspond to the social values of innovations in the green and grey sectors, respectively.

From now on we assume that, due to information and excludability problems, firms are unable to extract the whole willingnesses to pay for knowledge. We assume that they only extract a part $\bar{v}$, that corresponds to the market value of an innovation. In order to avoid heavy computations, we consider that extracted (i.e., market) values for one unit of knowledge are: $\bar{v}_{Q}=V_{Q} \delta_{Q} L_{Q}+\psi_{Q} l / A_{Q}$, and $\bar{v}_{R}=V_{R} \delta_{R} L_{R}+\psi_{R} \tau p_{R} R / A_{R}$, where $\psi_{i} \in[0 ; 1]$ for $i=Q, R$. This assumption can be interpreted as follows: innovators are able to fully observe the social value of innovations in the research activity, but not in the production activity. Moreover, in the following, we will interpret an increase in $\psi_{i}$ as an economic policy aiming at fostering one sector of research. Finally, unit prices paid for green and grey knowledge respectively are $V_{Q t}=\int_{t}^{+\infty} \bar{v}_{Q s} e^{-\int_{t}^{s} r_{u} d u} d s$ and $V_{R t}=$ $\int_{t}^{+\infty} \bar{v}_{R s} e^{-\int_{t}^{s} r_{u} d u} d s$. Differentiating, one gets the standard following formula:

$$
r_{t}=\frac{\dot{V}_{Q t}}{V_{Q t}}+\frac{\bar{v}_{Q t}}{V_{Q t}}=\frac{\dot{V}_{R t}}{V_{R t}}+\frac{\bar{v}_{R t}}{V_{R t}},
$$


which says that the rate of return is the same on the financial market as well as on the two research sectors.

Since we are in the symmetric case (in particular we have $Y_{n_{j}}=Y_{j} / N=Y / N$ and $p_{j}=p$ ), equation (21) becomes $p[1+(\varepsilon-1) / N]=\lambda$. Using (22) and (23), one gets

$$
p[1+(\varepsilon-1) / N]=p_{Q} Y^{\alpha-1} A_{Q}^{-\alpha} Q^{1-\alpha} / \beta=\tau p_{R} Y^{\alpha-1} A_{R}^{-\alpha} R^{1-\alpha} /(1-\beta)
$$

Since $\varepsilon<1$, this equation means that the price of any consumption good is higher than its marginal cost. Indeed, second and third terms represent the marginal costs when using the green and grey resources respectively. This gap between price and marginal cost allows firms to buy knowledge despite the non-convexity of technology. Observe that, if $N=1$ (monopolistic case), (29) becomes $p=$ (marginal cost) $/ \varepsilon$, which is the standard result.

Before we depict the general equilibrium in proposition 2, let us present a preliminary result.

Lemma: The following efficiency condition holds at each date $t$ :

$$
\frac{A_{Q t} Q_{t}}{A_{R t} R_{t}}=\left[\frac{\psi_{R}(1-\beta) \delta_{R}}{\psi_{Q} \beta \delta_{Q}}\right]^{1 / \alpha}
$$

This condition means that marginal productivity of labour is the same in both $R \& D$ sectors. Note that it holds at optimum also (see (41) below in Appendix 1).

Proof. See Appendix 2.

We now present the equilibrium of the decentralized economy in proposition $2^{7}$ :

Proposition 2 At the decentralized equilibrium, quantities and rates of growth take the

\footnotetext{
${ }^{7}$ As for the optimum, we have $E(0)=E_{0}, S(0)=S_{0}$ and $S_{0}=\int_{0}^{+\infty}\left(1+\mu_{t}\right) R_{t}^{e} d t$.
} 
following values (upper-script ${ }^{e}$ is used for equilibrium):

$$
\begin{gathered}
l^{e}=\frac{\rho}{\psi_{Q} \delta_{Q}}, Q^{e}=\theta l^{e}, \\
L_{Q}^{e}=\frac{\delta_{R}-\rho}{\delta_{Q}+\delta_{R}}-\frac{\rho \delta_{R}}{\psi_{Q} \delta_{Q}\left(\delta_{Q}+\delta_{R}\right)}-\frac{\hat{\mu}+g_{\tau}}{\delta_{Q}+\delta_{R}}, \\
L_{R}^{e}=\frac{\delta_{Q}+\rho}{\delta_{Q}+\delta_{R}}-\frac{\rho}{\psi_{Q}\left(\delta_{Q}+\delta_{R}\right)}+\frac{\hat{\mu}+g_{\tau}}{\delta_{Q}+\delta_{R}}, \\
E^{e}=E_{0}+\frac{\gamma R_{0}^{e}}{\rho+\hat{\mu}+g_{\tau}}\left[e^{-\left(\rho+\hat{\mu}+g_{\tau}\right) t}-1\right] . \\
g_{R}^{e}=-\rho-\hat{\mu}-g_{\tau}, \\
g_{Y}^{e}=\delta_{Q} L_{Q}^{e}, \\
g_{A_{Q}}^{e}=\delta_{Q} L_{Q}^{e}, g_{A_{R}}^{e}=\delta_{R} L_{R}^{e}, \text { and } g_{A_{Q}}^{e}-g_{A_{R}}^{e}=g_{R}^{e} .
\end{gathered}
$$

Proof. See Appendix 2.

Observe that the growth rate of the environmental tax has an impact on the equilibrium variables, whereas a change in the tax level only results in rent transfers (see also for instance Sinclair (1992), Nordhaus and Boyer (2000) and Grimaud and Rouge (2005)) ${ }^{8}$. Moreover, if $g_{\tau}$ is independant of time, and in particular if $g_{\tau}=0$, there are no transitional dynamics in the decentralized equilibrium; nevertheless, note that $E^{e}$ progressively decreases over time.

\subsubsection{Decentralized equilibrium vs. social optimum}

Assume that research is optimally funded $\left(\psi_{Q}=\psi_{R}=1\right)$ and that the optimal environmental policy is implemented, i.e. $g_{\tau}=g_{\tau}^{o}$ (the value of $g_{\tau}^{o}$ is given in Proposition 4

\footnotetext{
${ }^{8}$ Remark that no equilibrium variable depends on $\psi_{R}$. This can be explained as follows. Recall that $\psi_{R}$ only appears in the second term of the total willingness to pay for one unit of grey knowledge: see paragraph 3 in section 3.2. Basically, the equilibrium only determines the product $\psi_{R} \tau p_{R}$. Thus a change in $\psi_{R}$ has an effect which is similar to a change in the level of the environmental tax $\tau$, that is, only a rent transfer from the owner of the resource towards the government.
} 
below). Then, the equilibrium paths are similar to the optimal ones: all conditions given in Propositions 1 and 2 are identical, under the initial conditions $E(0)=E_{0}, S(0)=S_{0}$ and $S_{0}=\int_{0}^{+\infty}\left(1+\mu_{t}\right) R_{t}^{e} d t$. This means that all the variables of the model are identical at each date $t$ in the social planner regime and the decentralized economy. Observe that this holds despite the assumption of Cournot competition in differentiated goods' markets. Indeed, the mark-up in these markets entails a real wage lower than the walrasian one, but it does not prevent the optimum being reached. In this model there is only one representative household, who perceives two kinds of income: wages and profits. Since her labour supply is exogenous by assumption, when the real wage diminishes, total production is unchanged. Thus the household's total income is unchanged as the increase in her profits cancels the wage cut. So, her behavior in terms of consumption and savings is not modified and the general equilibrium of the economy is not affected. Barro and Sala-i-Martin (1995, Ch. 6, p. 234) obtain a similar result in the context of a monopoly pricing of consumer goods: in their context also, as labour supply is exogenous, the equilibrium is Pareto-optimal.

This underlines the fact that there are only three distortions preventing the economy achieving the social optimum: the possible inability of firms to extract the whole willingness to pay for knowledge in the two $R \& D$ sectors, and the environmental problem.

Let us now compare the equilibrium paths to the optimal ones. In Figure 2, equilibrium paths are represented by dashed lines, and these are shown with the optimal paths studied above (in section 2.2). Note that we assume $g_{\tau}=0$ and $\hat{\mu}=0$ (i.e., no environmental policy and constant extraction cost) in this figure. When $\psi_{Q}=1$, that is, when green research is fully funded, the only remaining distortion is the environmental one. Figure 2 shows that, in the long-run, the decentralized equilibrium tends to be socially optimal. Indeed, the environmental problem vanishes in the long-run since pollution flows tend to 
zero as the stock of resource is progressively exhausted.

Studying the depicted equilibrium paths shows the following, which complements the comments made in section 2.2. The equilibrium growth rate of resource extraction $\left(g_{R}^{e}=\right.$ $-\rho$ ) is too low with respect to the optimal rate: extraction (and thus pollution) has to be delayed, especially in early stages, when the gap is maximal. Labour devoted to green research $\left(L_{Q}^{e}\right)$ is too low (especially for the first generations here also); the lower $\psi_{Q}$ (increase in the $R \& D$ distortion), the bigger the gap is. Simultaneously, when $\psi_{Q}=1$, the effort invested in grey research $\left(L_{R}^{e}\right)$ is initially too high, and this over-investment in grey research tends to zero when $t$ tends to infinity. If $\psi_{Q}<1$, early generations overinvest in grey research (as previously), but future generations devote a sub-optimal effort to this research. Finally, output growth needs to be fostered (especially when there is a distortion in $R \& D$ sectors). Comments about the effects of economic policies given in section 4 provide further insight into these results.

Remark: Extraction costs $(\hat{\mu})$ have the same effect on equilibrium values as a change in $g_{\tau}$. In particular, if $\hat{\mu}$ is negative (technical progress), then equilibrium extraction is postponed. Indeed, because resource price increasing slows (see equation (20)), firms delay their use of this resource. We exhaustively describe the effects of a change in $g_{\tau}$ (and thus of $\hat{\mu}$ ) in the next section.

display Figure 2 here

\section{Economic policies}

\subsection{Impact of economic policy and direction of technical change}

Here we want to study the impact of an economic policy consisting of a subsidy to green research (an increase in $\psi_{Q}$ ) as well as an environmental policy (a change in $g_{\tau}$ ). 
Proposition 3 The effects of the economic policy are depicted in table 1.

\begin{tabular}{|c|c|c|c|c|c|c|c|c|c|}
\hline & $L_{Q}^{e}$ & $L_{R}^{e}$ & $l^{e}$ & $g_{R}^{e}$ & $g_{Y}^{e}=g_{A_{Q}}^{e}$ & $g_{A_{R}}^{e}$ & $g_{A_{Q}}^{e}-g_{A_{R}}^{e}$ & $g_{V_{Q}}^{e}$ & $g_{V_{R}}^{e}$ \\
\hline$\frac{\partial \cdot}{\partial \psi_{Q}}$ & $>0$ & $>0$ & $<0$ & $=0$ & $>0$ & $>0$ & $=0$ & & \\
\hline$\frac{\partial \cdot}{\partial g_{\tau}}$ & $<0$ & $>0$ & $=0$ & $<0$ & $<0$ & $>0$ & $<0$ & $<0$ & \\
\hline
\end{tabular}

Table 1: effects of economic policy

Proof. The results given in the first seven columns directly follow from formulas in proposition 2. The results in the last two columns are easily derived from (24) and (25), since one gets $g_{V_{Q}}^{e}=-g_{A_{Q}}^{e}$ and $g_{V_{R}}^{e}=-g_{A_{R}}^{e}$.

Let us now give some comments about results presented in proposition 3 .

\subsubsection{Effects of $R \& D$ policy}

An increase in $\psi_{Q}$, that is, an increase in the subsidy to green research leads to intuitive results. This reallocates labour among production $\left(l^{e}\right.$ decreases $)$ and research $\left(L_{Q}^{e}\right.$ and $L_{R}^{e}$ increase). Thus economic growth is fostered (since $\left.g_{Y}^{e}=g_{A_{Q}}^{e}=\delta_{Q} L_{Q}^{e}\right)$.

This policy has no effect on the extraction rate $\left(g_{R}^{e}\right.$ remains unchanged $)$. Since $g_{P}^{e}=g_{R}^{e}$, from (6), it has no effect on the rate of pollution emission either. As the pollution path is not modified, the environment is not affected ( $E_{t}$ remains unchanged for all $\left.t\right)$.

Recall that $g_{Y}^{e}=g_{A_{Q}}^{e}+g_{Q}^{e}=g_{A_{R}}^{e}+g_{R}^{e}$ (see (45) in Appendix 2). Since $g_{Q}^{e}=0$, we have $g_{A_{Q}}^{e}-g_{A_{R}}^{e}=g_{R}^{e}$. So, this economic policy fosters both research sectors, but it does not modify the direction of technical progress (i.e., $g_{A_{Q}}^{e}-g_{A_{R}}^{e}$ ). 


\subsubsection{Effects of environmental policy}

As we show in the next section, the optimal growth rate of the environmental tax is generally negative. For this reason, let us study the impact of a decrease in $g_{\tau}$, which we interpret as a more stringent environmental policy. We already know that a decreasing unit tax delays resource extraction (and thus polluting emissions) (see for instance Sinclair (1992)). We show in what follows that we obtain a similar result. Moreover, we prove that such a policy has environment friendly effects on the direction of technical change, namely, a rise in the growth rate of green knowledge, and a decrease in the growth rate of grey knowledge.

Impact on the paths of resource extraction and pollution emission Table 1 shows that a decrease in $g_{\tau}$ entails an increase in $g_{R}^{e}=g_{P}^{e}$. The mechanism can be described as follows.

Since $r=\rho$ (see Appendix 2), observe that $g_{\tau p_{R}}=g_{\tau}+r+\hat{\mu}$ decreases, that is, current values of $\tau p_{R}$ (the price paid by the consumption goods firms) increase relative to its future values. In other words, the non-renewable resource gets more expensive today and cheaper tomorrow. For this reason, the resource is extracted less rapidly (less today and more tomorrow): $g_{R}^{e}$ (and thus $g_{P}^{e}$ ) increases. Hence, resource extraction (and thus pollution emission) is delayed, as is the case in Sinclair (1992), Grimaud and Rouge (2005) and Groth and Schou (2007), for instance. Note that this first impact has a positive effect on output growth.

Impact on the direction and bias of technical change Table 1 shows that a decrease in $g_{\tau}$ entails an increase in $g_{A_{Q}}^{e}-g_{A_{R}}^{e}$. This results can be linked to Andre an Smulders (2004) (in a model without pollution). What follows gives intuitions about this result.

We have already shown that if $g_{\tau}$ decreases, then $g_{R}^{e}$ increases, that is, the extraction 
flow decreases in the short-run, and increases in the long-run. The initial decrease in $R^{e}$ entails a decrease in the marginal productivity of specific knowledge $A_{R}^{e}$. Indeed, $A_{R}^{e}$ and $R^{e}$ are complementary in the final sector (see comments below equation (1)); this implies that the rate of return in grey research $\left(\left((1-\beta) \psi_{R} \delta_{R} l / \beta\right)\left(A_{R}^{e} R^{e} / A_{Q}^{e} Q^{e}\right)^{\alpha}\right.$ : see proof of the Lemma in Appendix 2) decreases since it is an increasing function of $R^{e}$. Thus, investing in the grey $R \& D$ sector becomes less profitable, which yields a reallocation of labour among sectors. Less effort is devoted to the grey sector, and more to the green one: $L_{R}^{e}$ decreases and $L_{Q}^{e}$ increases (recall that $l$ is constant). Therefore, $g_{A_{R}}^{e}$ decreases and $g_{A_{Q}}^{e}$ increases. Finally, $g_{A_{Q}}^{e}-g_{A_{R}}^{e}$ increases, that is, technical progress is directed towards green knowledge.

Note that all these effects are reversed in the long run, as values of $R^{e}$ get higher in a distant future. However, the short-term effect outweights this long-term one because of intertemporal discounting.

In Table 1, a decrease in $g_{\tau}$ yields a decrease in $g_{V_{Q}}^{e}$ and an increase in $g_{V_{R}}^{e}$. We can give the following intuition about these equilibrium results. Since a more stringent environmental policy leads to an increase in $g_{A_{Q}}^{e}$, the unit cost of green innovations (i.e., $\left.\partial L_{Q} / \partial \dot{A}_{Q}=1 / \delta_{Q} A_{Q}\right)$ decreases faster. Profits being nil in research activities, the price of green innovations decreases faster also. The same argument applies to grey knowledge: a more stringent environmental policy entails a slower decrease in the unit cost of grey innovations, and thus in their prices also. Formally, from (24) and (25), one has $g_{V_{Q}}^{e}=$ $-g_{A_{Q}}^{e} \leq 0$ and $g_{V_{R}}^{e}=-g_{A_{R}}^{e} \leq 0$.

Finally, let us study the impact on the ratio of marginal productivities, noted $F_{Q} / F_{R}$, that we call the bias of technical change (in reference to Acemoglu (2002)). First, from (1), we have $F_{Q} / F_{R}=(\beta /(1-\beta))\left(A_{Q}^{e} / A_{R}^{e}\right)^{\alpha}\left(R^{e} / Q^{e}\right)^{1-\alpha}$

$$
=(\beta /(1-\beta))\left(A_{Q 0}^{e} / A_{R 0}^{e}\right)^{\alpha}\left(1 / Q^{e}\right)^{1-\alpha}\left(R_{0}^{e}\right)^{1-\alpha} \exp \left[\alpha\left(g_{A_{Q}}^{e}-g_{A_{R}}^{e}\right)+(1-\alpha) g_{R}^{e}\right] t
$$


$=(\beta /(1-\beta))\left(A_{Q 0}^{e} / A_{R 0}^{e}\right)^{\alpha}\left(1 / Q^{e}\right)^{1-\alpha}\left(R_{0}^{e}\right)^{1-\alpha} \exp g_{R}^{e} t$, since $g_{A_{Q}}^{e}-g_{A_{R}}^{e}=g_{R}^{e}$. A more stringent climate policy (a decrease in $g_{\tau}$ ) has different effects in the short and in the long-term. Indeed, it results in a decrease in $R_{0}^{e}$ and an increase in $\exp g_{R}^{e} t$ for $t>0$ (see above). When $t=0$, the only remaining effect is the former one. Thus, climate policy entails lower values of $F_{Q} / F_{R}$ : environmental policy is grey-biased in the short-term (this result holds in a neighborood of $t=0$ ). If $t$ is large, the latter effect dominates the former: $F_{Q} / F_{R}$ increases. Hence, environmental policy is green-biased in the long-run.

To sum up, we can classify the effects of the environmental policy into two types: growth and green effects.

Growth effects: A decrease in $g_{\tau}$ fosters the economy's growth: indeed positive impacts on $g_{R}^{e}$ and $g_{A_{Q}}^{e}$ in the same way yield an increase in $g_{Y}^{e}$. Note that, as we show in the following subsection, this means lower output levels for current generations and higher levels in the future.

Green effects: We distinguish two different green effects. First, $g_{R}^{e}$ increases: resource extraction, and thus pollution, is delayed. The second effect concerns the allocation of labour among the different $R \& D$ sectors of the economy. As we have seen, labour is transferred from the polluting resource $R \& D$ sector to the green one. This modifies the direction of technological change: it becomes more green-oriented.

\subsubsection{Utility levels}

The intertemporal utility of an economy shifting from a laissez-faire equilibrium to the social planner regime unambiguously increases. Nevertheless, the impact is not the same on all generations. Recall that the instantaneous utility function, $\ln \left(\int_{0}^{1} c_{j t}^{\varepsilon} d j\right)^{1 / \varepsilon}+\omega \ln E_{t}$ (see equation (9)), is an increasing function of the flow of consumption and the stock of environment; moreover consumption, i.e. production, is an increasing function of two 
inputs: $A_{Q} Q$ and $A_{R} R$ (see equation (1)).

Let us consider a laissez-faire equilibrium. Let us suppose that the economic policies presented above, namely an increase in $\psi_{Q}$ and a negative $g_{\tau}$, are implemented at date 0 . As $\psi_{Q}$ increases, the effort put into the production of the green resource, $l_{0}^{e}$, decreases (see Table 1). As a result, $Q_{O}^{e}=\theta l_{0}^{e}$ also decreases. Simultaneously, Table 1 shows that a decrease in $g_{\tau}$ yields an increase in $g_{R t}^{e}$ for all $t$. This results in a decrease in $R_{0}^{e}$. The stocks $E_{0}, A_{Q 0}$ and $A_{R 0}$ being unchanged, such economic policy unambiguously yields a decrease in $Y_{0}^{e}$. Henceforth, the instantaneous utility of the present generation diminishes. We can infer that the more the adjustment of the three stocks is progressive -which depens on the exogenous parameters of the model-, the more generations whose instantaneous utility decline following the first one will be numerous.

This means that the economic policy scheme presented here results in a loss of welfare for the first generations.

\subsection{Basic environmental externality and optimal policy}

Comparing values in propositions 1 and 2, we obtain the following result which gives the design of optimal policy instruments.

Proposition 4 If $\psi_{Q}=\psi_{R}=1$ (optimal financing of research) and $g_{\tau}=-\hat{\mu} B+$ $\frac{\omega\left(\delta_{Q}+\delta_{R}\right)}{\delta_{Q}} g_{E}^{o} \equiv g_{\tau}^{o}$, where $B=\left[\gamma\left(\delta_{Q}+\delta_{R}\right) R \int_{t}^{+\infty}(\omega / E) e^{-\rho(s-t)} d s\right] / \delta_{Q}$, then the decentralized equilibrium path is socially optimal.

A detailed interpretation of $g_{\tau}^{o}$ is given in Appendix 3. In what follows, we present the main intuitions.

The optimal rate of growth of the pollution tax, given in proposition 4 , is the social value of delaying an extraxted quantity corresponding to one unit of consumption good (i.e. $\left.\Delta R_{t}=-1 /\left(\partial Y_{t} / \partial R_{t}\right)\right)$, and thus delaying pollution, from $t$ to $t+\Delta t$ (that is, 
$\left.\Delta R_{t+\Delta t}=-\Delta R_{t}\right)$. This social value is expressed in terms of good $Y$, and in absolute value.

If $\hat{\mu}=0$ (constant unit cost of extraction), $g_{\tau}^{o}=\left[\omega\left(\delta_{Q}+\delta_{R}\right) / \delta_{Q}\right] g_{E}^{o}$, which is negative since $g_{E}^{o}$ is negative (see Proposition 1 and Figure 2): the optimal policy delays extraction (as in Sinclair (1992)). The basic mechanism is the following. Let us assume that extraction is reduced at time $t$, and that it is increased at $t+\Delta t$. Environment (and thus welfare) is not modified on the two intervals $]-\infty ; t[$ and $] t ;+\infty[$. On the reverse, it is improved on segment $[t ; t+\Delta t]$. That is the basic environmental externality of the model. Note that if we assumed environmental regeneration, or considered carbon sequestration, this would possibly change this result.

Note that Proposition 1 presents the first best. One could think of cases in which only a second best is achievable. For instance, $\psi_{Q}=\psi_{R}=1$ may be impossible, because of government resource constraints or observability issues, as in Gerlagh, Kverndokk and Rosendahl (2007). In this case, the optimal rate of growth of the environmental tax would differ from $g_{\tau}^{o}$ given in Proposition 4, and would explicitly depend on the research policy ${ }^{9}$. In Gerlagh, Kverndokk and Rosendahl, for instance, the optimal level of the environmental tax is higher than the Pigouvian level.

Remark: Let us consider the case where $\hat{\mu} \neq 0$. The term $\hat{\mu} B$ in $g_{\tau}^{o}$ is the change in utility for generations between $t$ and $+\infty$ if one delays extraction from $t$ to $t+\Delta t$. If $\hat{\mu}<0$, this utility decreases. Indeed, in this case, technical progress on extraction costs fosters extraction and thus pollution for future generations. This result has two contrary effects on the welfare of future generations: on the one hand, more extracted resource means more production. On the other hand, it also means more pollution. Here, technical progress is harmful in terms of environment for future generations. In the limit case $\hat{\mu}<\left[\omega\left(\delta_{Q}+\delta_{R}\right) / \delta_{Q} B\right] g_{E}^{o}$, which corresponds to a high level of technical progress

\footnotetext{
${ }^{9}$ We thank Reyer Gerlagh for this remark.
} 
in extraction, one gets $g_{\tau}^{o}>0$. Contrary to the standard view which recommends that resource extraction be postponed, we are here in the case in which extraction has to be accelerated.

\section{Conclusion}

We have presented an endogenous growth model in which consumption goods are produced by means of two inputs: a polluting non-renewable resource and a non-polluting labour resource. A specific research sector and a corresponding stock of knowledge are associated with each resource. The use of the non-renewable resource yields polluting emissions which damage the stock of environment, and this is harmful to household's utility. We have determined the social planner's optimal regime, which consists of a transition towards a stable unique optimal steady-state. We have also studied the properties of the economy's decentralized equilibrium, and have compared them to the optimal properties.

We have shown that the non-renewable resource is used too fast in the "laissez-faire" regime, and thus too much pollution is emitted in the early stages of the process. The overall research effort is lower than its optimal level; in fact, the effort put into green research is always too low, whilst that into grey research is too high in the early stages. Moreover, the direction of technical change is too 'grey-oriented' and the economy's growth is sub-optimal, which means that early generations consume too much.

We have studied the impact of three economic policy tools: two subsidies to research (green and grey) and an environmental tax on the non-renewable resource. Both types of research effort (green and grey) are fostered by the R\&D policy which, however, has no impact on the direction of technical change, on the flows of extraction (and thus of pollution), or the dynamics of the environment. The optimal environmental policy, which consists of a decreasing tax on fossil fuels, will hold back the pace of extraction, and 
thus slow down polluting emissions. This results from the fact that the price of the resource (including the tax) becomes relatively higher today. Furthermore, this policy fosters output growth, which entails a loss of welfare for early generations. Moreover, the environmental policy has no effect on the quantity of research but it modifies the quality of research: the effort put into 'grey' research decreases, thus benefiting 'green' research. We also showed that the environmental policy is grey-biased in the short-term, and greenbiased in the long-term. Finally, we determined the optimal values of the economic policy instruments employed.

Future lines of research could consider some cases in which the grey resource is not fully exhausted. One could also introduce regeneration into the law of motion of the environment. This, however, would modify the optimal trajectories and make interpretation and determination of the optimal tax more complex. Finally, considering carbon sequestration would allow us to dissociate resource extraction and polluting emissions. 


\section{Appendix}

\section{Appendix 1: Welfare}

Let us consider the symmetric case in which $N_{j}=N, Y_{n_{j}}=Y / N, Q_{n_{j}}=Q / N, R_{n_{j}}=$ $R / N, L_{Q n_{j}}=L_{Q} / N$, and $L_{R n_{j}}=L_{R} / N$. Then technologies (1), (2) and (3) become $Y=\left[\beta\left(A_{Q} Q\right)^{\alpha}+(1-\beta)\left(A_{R} R\right)^{a}\right]^{1 / \alpha}, \dot{A}_{Q}=\delta_{Q} L_{Q} A_{Q}$, and $\dot{A}_{R}=\delta_{R} L_{R} A_{R}$. Utility is now $U=\int_{0}^{+\infty}\left(\ln c_{t}+\omega \ln E_{t}\right) e^{-\rho t} d t$. The social planner maximizes $U$ subject to the modified versions of (1), (2) and (3), and (4), (5), (6), (7) and (8). The Hamiltonian of the program is

$$
\begin{aligned}
H= & \left\{\frac{1}{\alpha} \ln \left[\beta\left(A_{Q} \theta l\right)^{\alpha}+(1-\beta)\left(A_{R} R\right)^{a}\right]+\omega \ln E\right\} e^{-\rho t}+\varphi_{Q} \delta_{Q}\left(1-l-L_{R}\right) A_{Q} \\
& +\varphi_{R} \delta_{R} L_{R} A_{R}-\varphi_{S}(1+\mu) R-\varphi_{E} \gamma R .
\end{aligned}
$$

The first order conditions $\partial H / \partial l=0, \partial H / \partial L_{R}=0$ and $\partial H / \partial R=0$ yield

$$
\begin{gathered}
Y^{-\alpha} \beta \theta^{\alpha} A_{Q}^{\alpha} l^{\alpha-1} e^{-\rho t}-\varphi_{Q} \delta_{Q} A_{Q}=0 \\
-\varphi_{Q} \delta_{Q} A_{Q}+\varphi_{R} \delta_{R} A_{R}=0 \\
Y^{-\alpha}(1-\beta) A_{R}^{\alpha} R^{\alpha-1} e^{-\rho t}-\varphi_{S}(1+\mu)-\varphi_{E} \gamma=0 .
\end{gathered}
$$

Moreover, $\partial H / \partial A_{Q}=-\dot{\varphi}_{Q}, \partial H / \partial A_{R}=-\dot{\varphi}_{R}, \partial H / \partial S=-\dot{\varphi}_{S}$, and $\partial H / \partial E=-\dot{\varphi}_{E}$ yield

$$
\begin{gathered}
\frac{\dot{\varphi}_{Q}}{\varphi_{Q}}=\frac{-Y^{-\alpha} \beta(\theta l)^{\alpha} A_{Q}^{\alpha-1} e^{-\rho t}}{\varphi_{Q}}-\delta_{Q}\left(1-l-L_{R}\right), \\
\frac{\dot{\varphi}_{R}}{\varphi_{R}}=\frac{-Y^{-\alpha}(1-\beta) R^{\alpha} A_{R}^{\alpha-1} e^{-\rho t}}{\varphi_{R}}-\delta_{R} L_{R},
\end{gathered}
$$




$$
\begin{gathered}
-\dot{\varphi}_{S}=0, \\
\text { and } \frac{\omega}{E} e^{-\rho t}=-\dot{\varphi}_{E} .
\end{gathered}
$$

i) Efficiency and Ramsey-Keynes conditions:

Replacing $\varphi_{Q}$ and $\varphi_{R}$ in (34) and (35) by their expressions in (31) and (32) gives

$$
g_{\varphi_{Q}}=-\delta_{Q}\left(l+L_{Q}\right)
$$

and

$$
g_{\varphi_{R}}=\frac{-(1-\beta) \delta_{R} A_{R}^{\alpha} R^{\alpha}}{\beta \theta^{\alpha} A_{Q}^{\alpha} l^{\alpha-1}}-\delta_{R} L_{R}
$$

Log-differentiating (31) with respect to time yields $-\alpha g_{Y}+\alpha g_{A_{Q}}+(\alpha-1) g_{l}-\rho=g_{\varphi_{Q}}+g_{A_{Q}}$, which, together with (38) yields Ramsey-Keynes condition

$$
\rho=-\alpha g_{Y}+\alpha g_{A_{Q}}+(\alpha-1) g_{l}+\delta_{Q} l
$$

Log-differentiating (32) with respect to time yields $g_{\varphi_{Q}}+g_{A_{Q}}=g_{\varphi_{R}}+g_{A_{R}}$, which, together with (38), (39) and (4) yields

$$
\frac{A_{Q} Q}{A_{R} R}=\left[\frac{(1-\beta) \delta_{R}}{\beta \delta_{Q}}\right]^{1 / \alpha}
$$

which is an efficiency condition saying that marginal productivity of labour in both research sectors is the same. For a similar condition, see condition (20) in Acemoglu (2002).

ii) Hotelling condition:

Log-differentiating (33) with respect to time, we get $-\alpha g_{Y}+\alpha g_{A_{R}}+(\alpha-1) g_{R}-\rho=$ $\frac{\left(\varphi_{S}(\dot{1}+\mu)\right)+\dot{\varphi}_{E} \gamma}{(1-\beta) Y^{-\alpha} A_{R}^{\alpha} R^{\alpha-1} e^{-\rho t}}$. Observe that $\left(\varphi_{S}(\dot{1}+\mu)\right)=\varphi_{S} \dot{\mu}$ since $\dot{\varphi}_{S}=0$, from (36). Replacing 
$\varphi_{S}$ and $\dot{\varphi}_{E}$ by their expressions in (33) and (37), we get

$-\alpha g_{Y}+\alpha g_{A_{R}}+(\alpha-1) g_{R}-\rho=\hat{\mu}\left[1-\frac{\varphi_{E} \gamma}{(1-\beta)\left(A_{R} R / Y\right)^{\alpha} R^{-1} e^{-\rho t}}\right]-\frac{\gamma \omega}{(1-\beta)\left(A_{R} R / Y\right)^{\alpha} R^{-1} E}$.

First, since $Y=A_{R} R\left[\beta\left(A_{Q} Q / A_{R} R\right)^{\alpha}+(1-\beta)\right]^{1 / \alpha}$ (which is (1) in the symmetric case), and $A_{Q} Q / A_{R} R=\left[(1-\beta) \delta_{R} / \beta \delta_{Q}\right]^{1 / \alpha}$ (see $\left.(41)\right)$, we have $(1-\beta)\left(A_{R} R / Y\right)^{\alpha}=$ $\delta_{Q} /\left(\delta_{Q}+\delta_{R}\right)$. Moreover, we have $g_{Y}=g_{A_{R}}+g_{R}$. This allows us to write $-\alpha g_{Y}+\alpha g_{A_{R}}+$ $(\alpha-1) g_{R}-\rho=-g_{R}-\rho$.

Second, integrating (37) gives $\varphi_{E}=\varphi_{E 0}-\int_{0}^{t} \frac{\omega}{E} e^{-\rho s} d s$. Transversality condition $\lim _{t \rightarrow+\infty} \varphi_{E} E=0$ becomes $\lim _{t \rightarrow+\infty}\left(\varphi_{E 0}-\int_{0}^{t} \frac{\omega}{E} e^{-\rho s} d s\right)\left(E_{0}-\int_{0}^{t} \gamma R_{s} d s\right)=0$. The second term between brackets tends to a finite limit (which we can assume different from zero) since the integral is finite (the stock of resource being finite). Thus $\varphi_{E 0}=\int_{0}^{+\infty} \frac{\omega}{E} e^{-\rho s} d s$, which gives $\varphi_{E}=\int_{t}^{+\infty} \frac{\omega}{E} e^{-\rho s} d s$.

Third, from $\dot{E}=-\gamma R$ we have $g_{E}=-\gamma R / E$.

Finally, plugging these results into (42) yields the following Hotelling rule

$$
g_{R}=-\rho-\hat{\mu}\left(1-\frac{\gamma\left(\delta_{Q}+\delta_{R}\right) R}{\delta_{Q}} \int_{t}^{+\infty}(\omega / E) e^{-\rho(s-t)} d s\right)-\frac{\omega\left(\delta_{Q}+\delta_{R}\right)}{\delta_{Q}} g_{E}
$$

iii) Computation of the optimal solutions:

Computation of $l^{o}$. Since $A_{Q} Q / A_{R} R$ is constant (from (41)), we have $\delta_{Q} L_{Q}+g_{Q}=$ $\delta_{R} L_{R}+g_{R}$. However, we have already proved that $g_{Y}=\delta_{R} L_{R}+g_{R}$ (see ii) above). Thus, $g_{Y}=\delta_{Q} L_{Q}+g_{Q}$. Plugging this into (40) and using $g_{Q}=g_{l}$, we obtain the following Ricatti differential equation: $i=\delta_{Q} l^{2}-\rho l$. In order to transform this equation into a linear first-order differential equation, we consider the new variable $z=1 / l$, which implies $\dot{z}=-i / l^{2}$. The Ricatti equation becomes $\dot{z}=-\delta_{Q}+\rho z$, whose solution leads to $l=\frac{1}{e^{\rho t}\left(1 / l_{0}-\delta_{Q} / \rho\right)+\delta_{Q} / \rho}$. Using transversality condition $\lim _{t \longrightarrow+\infty} \varphi_{Q} A_{Q}=0$, we show that $l$ 
immediately jumps to its steady-state level:

$$
l^{o}=\rho / \delta_{Q}
$$

Indeed, with formula (31), we get $\varphi_{Q} A_{Q}=\frac{\beta}{\delta_{Q}}\left(\frac{A_{Q} Q}{Y}\right)^{\alpha} e^{-\rho t}\left[e^{\rho t}\left(\frac{1}{l_{0}}-\frac{\delta_{Q}}{\rho}\right)+\frac{\delta_{Q}}{\rho}\right]$, where $A_{Q} Q / Y$ is constant. It turns out that transversality condititon $\lim _{t \rightarrow+\infty} \varphi_{Q} A_{Q}=0$ is only satisfied when $l_{0}=\rho / \delta_{Q}$.

Computation of $L_{Q}^{o}, L_{R}^{o}$ and $g_{Y}^{o}$. From (8), we know that $L_{R}^{o}=1-l^{o}-L_{Q}^{o}$. Moreover, we have already seen that $\delta_{Q} L_{Q}+g_{Q}=\delta_{R} L_{R}+g_{R}$. Taking into account the fact that $l^{o}$ is a constant, we can easily express $L_{Q}^{o}$ in terms of $g_{R}^{o}$. We obtain formula (11). Then we can compute $L_{R}^{o}$ in terms of $g_{R}^{o}$ (see (12)). Since $g_{Y}=\delta_{Q} L_{Q}+g_{Q}$, the expression of $g_{Y}^{o}$ follows (see (14)).

\section{Appendix 2: Equilibrium}

- Household's behaviour.

The current value Hamiltonian of the household's programme is

$H=(1 / \varepsilon) \ln \int_{0}^{1} c_{j}^{\varepsilon} d j+\omega \ln E+\mu\left(r b+w+\pi-\int_{0}^{1} p_{j} c_{j} d j+T\right)$, where $\mu$ is the co-state variable associated with $b$. The two conditions $\partial H / \partial c_{j}=0$ and $\partial H / \partial b=\rho \mu-\dot{\mu}$ lead to $\mu=c_{j}^{\varepsilon-1} / \Gamma p_{j}$, where $\Gamma=\int_{0}^{1} c_{j}^{\varepsilon} d j$, and $\dot{\mu} / \mu=\rho-r$.

From the first condition, one gets $c_{j}^{\varepsilon-1} / c_{k}^{\varepsilon-1}=p_{j} / p_{k}$, for all $j, k$. Multiplying both sides by $p_{k}^{1-\varepsilon}$ and rearranging, we obtain $p_{k}^{-\varepsilon /(1-\varepsilon)} p_{j}^{1 /(1-\varepsilon)} c_{j}=p_{k} c_{k}$. Summing on $k$ finally gives $c_{j}=\left[p_{j}^{1 /(\varepsilon-1)} \int_{0}^{1} p_{k} c_{k} d k\right] / \int_{0}^{1} p_{k}^{\varepsilon /(\varepsilon-1)} d k$.

Differentiating the first condition with respect to time gives $g_{\mu}=(\varepsilon-1) g_{c_{j}}-g_{\Gamma}-g_{p_{j}}$. Together with the second condition, this gives the following Ramsey-Keynes condition: $r=\rho+(1-\varepsilon) g_{c_{j}}+g_{\Gamma}+g_{p_{j}}$

- Proof of the lemma. 
From (24) we have $g_{V_{Q}}=-g_{A_{Q}}=-\delta_{Q} L_{Q}$. Then, using (28), where $\bar{v}_{Q}=V_{Q} \delta_{Q} L_{Q}+$ $\psi_{Q} l / A_{Q}$, one gets $r=\psi_{Q} \delta_{Q} l$ (which is the rate of return in green research).

Similarly, from (25) we obtain $g_{V_{R}}=-g_{A_{R}}=-\delta_{R} L_{R}$. Formula (28), with $\bar{v}_{R}=$ $V_{R} \delta_{R} L_{R}+\psi_{R} \tau p_{R} R / A_{R}$, yields $r=\psi_{R} \tau p_{R} \delta_{R} R$. Using the expression of $\tau p_{R}$ given by (29), $p_{Q}=1 / \theta($ see $(19))$, and $Q=\theta l$ (see (4)), we obtain $\tau p_{R}=((1-\beta) l / \beta R)\left(A_{R} R / A_{Q} Q\right)^{\alpha}$. Plugging this formula in the expression of $r$, we get $r=\left((1-\beta) \psi_{R} \delta_{R} l / \beta\right)\left(A_{R} R / A_{Q} Q\right)^{\alpha}$. This is the rate of return in grey research.

Thus, we have two expressions of $r$, which allow us to get condition (30).

- Two preliminary results.

Technology $Y=\left[\beta\left(A_{Q} Q\right)^{\alpha}+(1-\beta)\left(A_{R} R\right)^{\alpha}\right]^{1 / \alpha}$ (which is (1) in the symmetric case) can be written $Y=A_{Q} Q\left[\beta+(1-\beta)\left(A_{Q} Q / A_{R} R\right)^{-\alpha}\right]^{1 / \alpha}=A_{R} R\left[\beta z^{\alpha}+(1-\beta)\right]^{1 / \alpha}$. Since $A_{Q} Q / A_{R} R$ is constant from lemma 1 , log-differentiation with respect to time gives

$$
g_{Y}=g_{A_{Q}}+g_{Q}=g_{A_{R}}+g_{R}
$$

Second, from (29), we have (observe that, from (19), $g_{p_{Q}}=0$ )

$$
g_{p}=(\alpha-1) g_{Y}-\alpha g_{A_{Q}}+(1-\alpha) g_{Q}=g_{\tau}+g_{p_{R}}+(\alpha-1) g_{Y}-\alpha g_{A_{R}}+(1-\alpha) g_{R}
$$

- Determination of $l$.

In the symmetric case, we have $\Gamma=\varepsilon g_{c}$. Thus, Ramsey-Keynes condition (18) becomes $r=\rho+g_{c}+g_{p}$. Let us replace $g_{p}$ by $(\alpha-1) g_{Y}-\alpha g_{A_{Q}}+(1-\alpha) g_{Q}$ (see equation $\left.(46)\right)$ and recall that $g_{Y}=g_{c}, g_{Y}-g_{A_{Q}}=g_{Q}=g_{l}$ (see (45) and (4)) and $r=\psi_{Q} \delta_{Q} l$ (see proof of lemma 1). We obtain $\rho+g_{l}=\psi_{Q} \delta_{Q} l$, that is, $i=\psi_{Q} \delta_{Q} l^{2}-\rho l$, which is a Ricatti differential equation. In order to transform this equation into a linear first-order differential equation, we consider the new variable $z=1 / l$, which implies $\dot{z}=-i / l^{2}$. The Ricatti equation 
becomes $\dot{z}=-\psi_{Q} \delta_{Q}+\rho z$, whose solution leads to $l=\frac{1}{e^{\rho t}\left(1 / l_{0}-\psi_{Q} \delta_{Q} / \rho\right)+\psi_{Q} \delta_{Q} / \rho}$. Using the transversality condition of the household's program, we can show, as we did in Appendix 1 , that $l$ immediately jumps to its steady-state level. Thus, one gets $l=\rho / \psi_{Q} \delta_{Q}$.

Note that we also obtain $r=\rho$ : this result come from the fact that we normalized wage to one (see section 3.1). If we had normalized the price of consumption goods to one in symmetric decentralized equilibrium, the interest rate would have been equal to $\rho+g_{Y}$ (which is a more conventional finding).

- Determination of $g_{R}$.

Log-differentiating (29) with respect to time, and using (20) gives $g_{p}=g_{\tau}+\hat{\mu}+r+$ $(\alpha-1) g_{Y}-\alpha g_{A_{R}}+(1-\alpha) g_{R}$. Plugging this expression in (18), and since $g_{Y}-g_{A_{R}}=g_{R}$, one gets $g_{R}=-\rho-\hat{\mu}-g_{\tau}$.

- Labour in $R \& D$.

From (45), and $g_{Q}=g_{l}=0$, we have $\delta_{Q} L_{Q}=\delta_{R} L_{R}+g_{R}$. Since $L_{R}=1-L_{Q}-\rho / \psi_{Q} \delta_{Q}$, one gets $L_{Q}=\frac{\delta_{R}-\rho}{\delta_{Q}+\delta_{R}}-\frac{\rho \delta_{R}}{\psi_{Q} \delta_{Q}\left(\delta_{Q}+\delta_{R}\right)}-\frac{\hat{\mu}+g_{\tau}}{\delta_{Q}+\delta_{R}}$. Then we obtain $L_{R}^{e}=\frac{\delta_{Q}+\rho}{\delta_{Q}+\delta_{R}}-\frac{\rho}{\psi_{Q}\left(\delta_{Q}+\delta_{R}\right)}+$ $\frac{\hat{\mu}+g_{\tau}}{\delta_{Q}+\delta_{R}}$.

- Growth of output.

From (45), we have $g_{Y}=g_{A_{Q}}=\delta_{Q} L_{Q}$.

- Stock of environment.

From $E_{t}=E_{0}-\int_{0}^{t} \gamma R_{s} d_{s}$, and $g_{R}=-\rho-\hat{\mu}-g_{\tau}$, one gets $E_{t}=E_{0}-\int_{0}^{t} \gamma R_{0} e^{-\left(\rho+\hat{\mu}+g_{\tau}\right) s} d s=$ $E_{0}+\frac{\gamma R_{0}}{\rho+\hat{\mu}+g_{\tau}}\left[e^{-\left(\rho+\hat{\mu}+g_{\tau}\right) t}-1\right]$. After differentiation, we obtain the rate of growth of this stock : $g_{E}=\frac{1}{e^{\left(\rho+\hat{\mu}+g_{\tau}\right) t}\left[1 / g_{E 0}+1 /\left(\rho+\hat{\mu}+g_{\tau}\right)\right]-1 /\left(\rho+\hat{\mu}+g_{\tau}\right)}$.

\section{Appendix 3: Optimal environmental policy}

- Preliminary result: marginal productivity of the non-renewable resource.

$\begin{array}{llll}\text { From } & (1) \quad \text { and } & \text { we }\end{array}$ 
$Y_{t}=A_{R t} R_{t}\left[\beta\left(A_{Q t} Q_{t} / A_{R t} R_{t}\right)^{\alpha}+(1-\beta)^{\alpha}\right]^{1 / \alpha}=A_{R t} R_{t}\left[(1-\beta) \delta_{R} / \delta_{Q}+1-\beta\right]^{1 / \alpha}$

Marginal productivity of the resource is $\partial Y_{t} / \partial R_{t}=(1-\beta) Y_{t}^{1-\alpha} A_{R t}^{\alpha} R_{t}^{\alpha-1}=(1-$ $\beta)\left(A_{R t} R_{t} / Y_{t}\right)^{\alpha}\left(Y_{t} / R_{t}\right)$. Using the previous result, one gets $\partial Y_{t} / \partial R_{t}=\left[\delta_{Q} /\left(\delta_{Q}+\delta_{R}\right)\right]\left(Y_{t} / R_{t}\right)$.

- Assume $\Delta C_{t}=-1$, which corresponds to a decrease in extraction $\Delta R_{t}=-1 /\left(\partial Y_{t} / \partial R_{t}\right)=$ $\left[-\left(\delta_{Q}+\delta_{R}\right) / \delta_{Q}\right]\left(R_{t} / Y_{t}\right)$. Suppose that this extraction is delayed until $t+\Delta t$, we have $\Delta R_{t+\Delta t}=-\Delta R_{t}=\left[\left(\delta_{Q}+\delta_{R}\right) / \delta_{Q}\right]\left(R_{t} / Y_{t}\right)$

- Impact on the environment.

First, remember that $E_{t}=E_{0}-\int_{0}^{t} P_{s} d_{s}$. Between $t$ and $t+\Delta t$, environmental quality is improved: $d E_{t, t+\Delta t}=\left[\gamma\left(\delta_{Q}+\delta_{R}\right) / \delta_{Q}\right]\left(R_{t} / Y_{t}\right)$. For any $s \in[t+\Delta t,+\infty[$, environmental quality is modified according to $d E_{s}=-\hat{\mu}\left[\gamma\left(\delta_{Q}+\delta_{R}\right) / \delta_{Q}\right]\left(R_{s} / Y_{s}\right)$. If $\hat{\mu}>0$, this is harmful for environment, and if $\hat{\mu}<0$, this improves environmental quality.

- Social value of delaying extraction.

A change $d E_{t}$ corresponds to the following change in consumption (i.e., the marginal rate of substitution): $\quad d C_{t}=[-(\partial U / \partial E) /(\partial U / \partial C)] d E_{t}$ $=\left[-\left(\omega / E_{t}\right) /\left(1 / C_{t}\right)\right] d E_{t}=\left(-\omega C_{t} / E_{t}\right) d E_{t}$.

On the interval $[t, t+\Delta t]$, we get $d C_{t, t+\Delta t}=\left(-\omega C_{t} / E_{t}\right) d E_{t, t+\Delta t}$ $=\left(-\omega C_{t} / E_{t}\right)\left[\gamma\left(\delta_{Q}+\delta_{R}\right) / \delta_{Q}\right]\left(R_{t} / Y_{t}\right)=\left[-\omega \gamma\left(\delta_{Q}+\delta_{R}\right) / \delta_{Q}\right]\left(R_{t} / E_{t}\right)$. From (7), we have $\gamma R_{t} / E_{t}=-g_{E}$. Finally, we obtain $d C_{t, t+\Delta t}=\left[\omega\left(\delta_{Q}+\delta_{R}\right) / \delta_{Q}\right] g_{E}$.

On the interval $[t+\Delta t,+\infty[$, the total variation of utility is given by $-\hat{\mu}\left[\gamma\left(\delta_{Q}+\delta_{R}\right) / \delta_{Q}\right]\left(R_{t} / Y_{t}\right) \int_{t+\Delta t}^{+\infty}\left(\omega / E_{s}\right) e^{-\rho(s-t)} d s$. Since $\partial U / \partial C_{t}=1 / C_{t}=1 / Y_{t}$, the corresponding change in consumption is $d C_{t+\Delta t,+\infty}=-\hat{\mu}\left[\gamma\left(\delta_{Q}+\delta_{R}\right) / \delta_{Q}\right] R_{t} \int_{t+\Delta t}^{+\infty}\left(\omega / E_{s}\right) e^{-\rho(s-t)} d s$.

Observe that $\quad d C_{t, t+\Delta t}+t_{t+\Delta t,+\infty}$ $=\left[\omega\left(\delta_{Q}+\delta_{R}\right) / \delta_{Q}\right] g_{E}-\hat{\mu}\left[\gamma\left(\delta_{Q}+\delta_{R}\right) / \delta_{Q}\right] R_{t} \int_{t+\Delta t}^{+\infty}\left(\omega / E_{s}\right) e^{-\rho(s-t)} d s$, that is (assuming that $\Delta t \rightarrow 0$ ), the optimal growth rate of the environmental tax given in proposition 4. 


\section{References}

Acemoglu D (2002) Directed technical change. Review of Economic Studies 69:781-809

André FJ, Smulders S (2004) Energy use, endogenous technical change and economic growth. Mimeo

Barro RJ, Sala-i-Martin X (1995) Economic Growth. McGraw-Hill, Inc

Chakravorty U, Roumasset J, Tse K (1997) Endogenous Substitution of Energy Resources and Global Warming. Journal of Political Economy 105(6):1201-1234

Dasgupta PS, Heal GM (1974) The optimal depletion of exhaustible resources. Review of Economic Studies. Symposium, 41: 3-28

Dasgupta PS, Heal GM (1979) Economic theory and exhaustible resources. Cambridge Economic Handbooks

Edenhofer 0, Bauer N, Kriegler E (2005) The impact of technological change on climate protection and welfare: Insights from the model MIND. Ecological Economics 54(2-3):277292

Gerlagh R, Kverndokk S, Rosendahl KE (2007) Optimal Timing of Environmental Policy (Interaction between Environmental Taxes and Innovation Externalities). Statistics Norway, Research Department. Discussion Papers 493

Gerlagh R, Keyzer MA (2004) Path-dependence in a Ramsey model with resource amenities and limited regeneration. Journal of Economic Dynamics \& Control 28:11591184

Gerlagh R, Lise W (2005) Carbon taxes: A drop in the ocean, or a drop that erodes the stone? The effect of carbon taxes on technological change. Ecological Economics $54(2-3): 241-260$

Gerlagh R, van der Zwaan B (2003) Gross world product and consumption in a global warming model with endogenous technological change. Resource and Energy Economics 
$25: 35-57$

Grimaud A, Rouge L (2004) Polluting non-renewable resources, tradeable permits and endogenous growth. International Journal of Global Environmental Issues 4:38-57

Grimaud A, Rouge L (2005) Polluting Non-Renewable Resources, Innovation and Growth: Welfare and Environmental Policy. Resource and Energy Economics 27(2):109129

Groth C, Schou P (2007) Growth and Non-Renewable Resources: The Different Roles of Capital and Resource Taxes. Journal of Environmental Economics and Management $53: 80-98$

Hart R (2004) Growth, Environment annd Innovation - a Model with Production Vintages and Environmentally Oriented Research, Journal of Environmental Economics and Management 48:1078-1098

Hoel M, Kverndokk S (1996) Depletion of Fossil Fuels and the Impacts of Global Warming. Resource and Energy Economics 18:115-136

Jones CI, Williams JC (1998) Measuring the Social Returns to R\&D. Quaterly Journal of Economics 113:1119-1135

Liski M, Tahvonen O (2004) Can carbon tax eat OPEC's rents? Journal of Environmental Economics and Management 47:1-12

Nordhaus WD, Boyer J (2000) Warming the World: Economic Models of Global Warming. MIT Press

Popp D (2004) ENTICE: Endogenous technological change in the DICE model of global warming. Journal of Environmental Economics and Management 48:742-768

Popp D (2006) ENTICE-BR: The effects of backstop technology R\&D on climate policy models. Energy Economics, Forthcoming

Schou P (2000) Polluting non-renewable resources and growth. Environmental and 
Resource Economics 16:211-227

Schou P (2002) When environmental policy is superfluous: growth and polluting resources. Scandinavian Journal of Economics 104:605-620

Sinclair P (1992) High does nothing and rising is worse: carbon taxes should keep declining to cut harmful emissions. The Manchester School 60(1):41-52 .

Smulders S, de Nooij M (2003) The Impact of Energy Conservation on Technology and Economic Growth. Resource and Energy Economics 25:59-79

Tahvonen O (1997) Fossil Fuels, Stock Externalities, and Backstop Technology. Canadian Journal of Economics XXX 4a:855-874 .

Tahvonen O, Salo S (2001) Economic Growth and Transition Between Renewable and Nonrenewable Energy Resources. European Economic Review 45:1379-1398

Tinbergen T (1960) On the Theory of Economic Policy. Elsevier, Amsterdam

Ulph A, Ulph D (1994) The Optimal Time Path of a Carbon Tax. Oxford Economic papers $46: 857-868$

Withagen C (1994) Pollution and Exhaustibility of fossil fuels. Resource and Energy Economics 16:235-242 


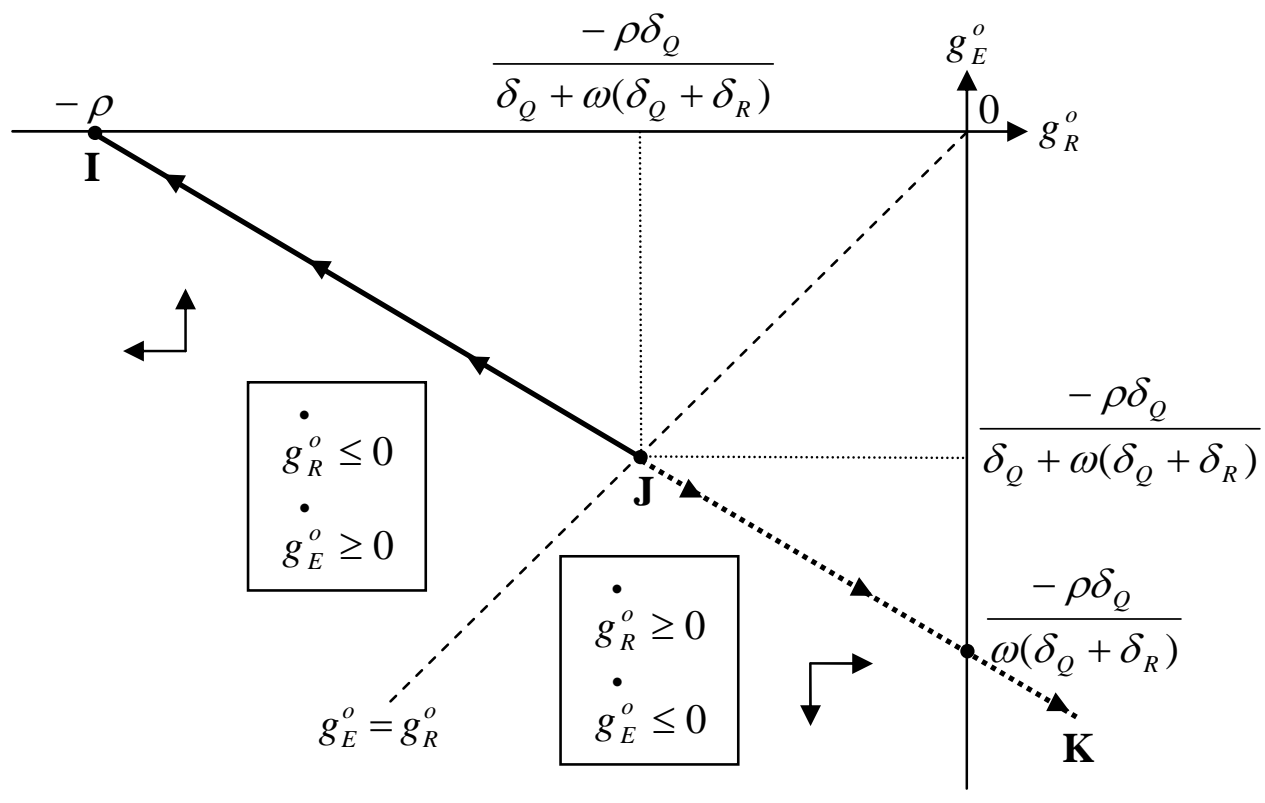

Figure 1: Phase Diagram 

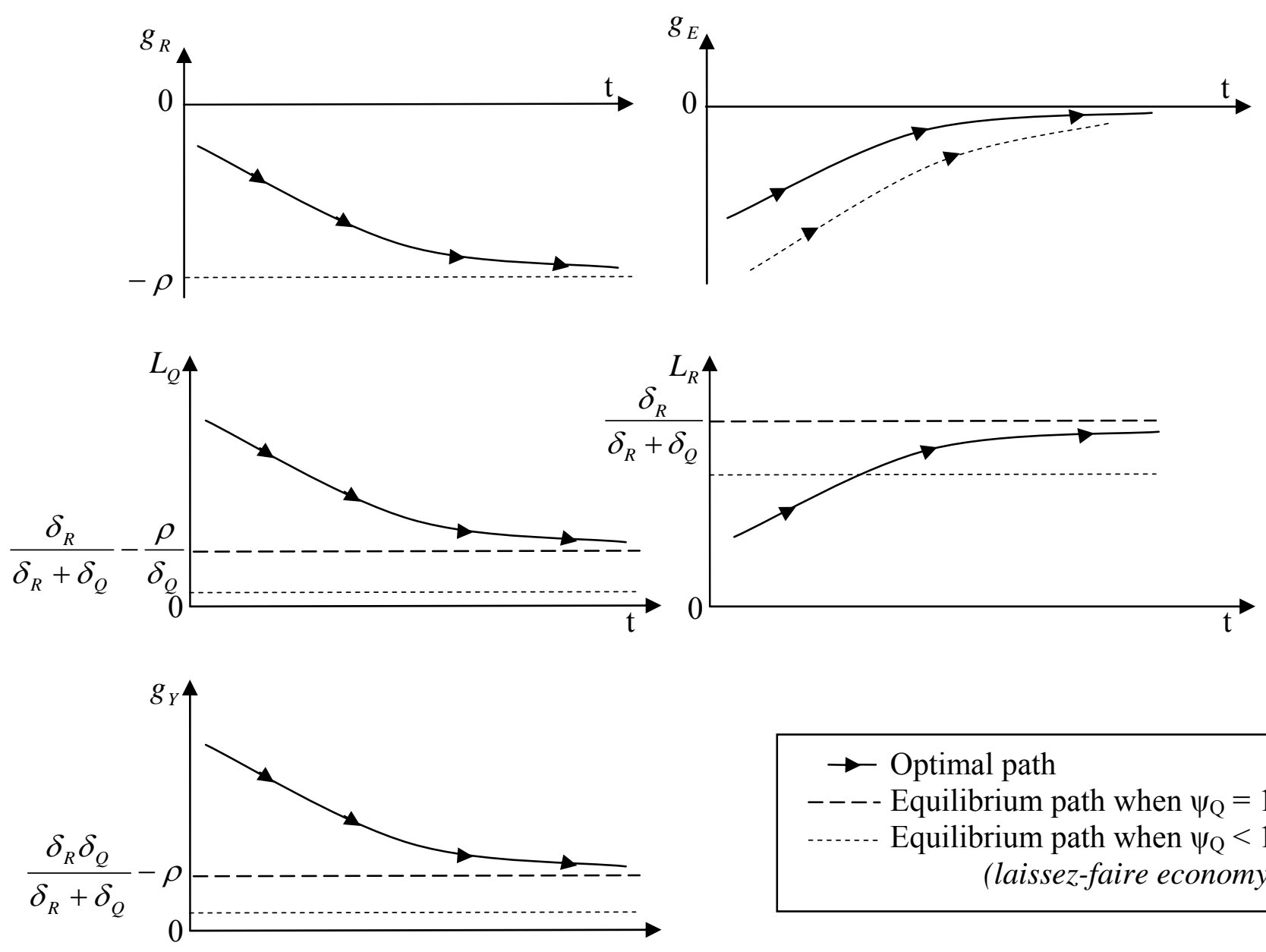

$\rightarrow$ Optimal path

- - - Equilibrium path when $\psi_{\mathrm{Q}}=1$ Equilibrium path when $\psi_{\mathrm{Q}}<1$

(laissez-faire economy)

Figure 2: Dynamics 